\title{
Recommandations, consensus, agences de santé ou instituts nationaux et conflits d'intérêt : quelles relations ou coopérations en 2012 ?
}

\section{Recommendations, consensus, healthcare agencies or national institutes and conflicts of interest: what collaborations or partnerships will there be in 2012 ?}

\section{Heresbach}

(C) Springer-Verlag France 2011

L'exercice de notre spécialité est encadré, d'une part, par une cotation dont « l'équilibre » est assuré, par les discussions et arbitrages conventionnels - dont les acteurs sont essentiellement le couple Ucam-Syndicat — et, d'autre part, par les recommandations de bonne pratique et codification, dont l'aboutissement est un échange entre les associations professionnelles, généralement les sociétés savantes et les agences nationales de santé. Signe des temps, le concept " coûtefficacité » s'est invité dans le débat, mais son positionnement inattendu ne devrait pas parasiter certaines décisions... sauf à connaître à l'avance les conflits d'intérêt également de ceux qui rendent ces décisions de refus ou d'acceptation des saisines formulées par les professionnels de santé. Ainsi, pendant l'année 2011, la commission " recommandation » de la SFED a observé un comportement étrange de nos interlocuteurs institutionnels en particulier de la HAS et l'INCa.

Alors qu'une réflexion sur la forme et les thèmes que la commission " recommandation » doit aborder en complément des sujets déjà traités par d'autres commissions de la SFED ou d'autres sociétés savantes, le thème de la « prise en charge des polypes rectocoliques de moins de $5 \mathrm{~mm} » \mathrm{~s}$ 'est naturellement imposé. En effet, alors que le choix des marqueurs de qualité de la coloscopie, taux de détection des adénomes ou incidence des cancers d'intervalle, est débattu, déposer chaque année à la HAS un dossier de saisine relève des missions et compétences de la SFED. Cette demande était d'autant plus motivée que parmi les conclusions du dossier, déjà sur saisine de la HAS par la SFED (revue technologique de la coloscopie virtuelle réalisée en 2010) figurait « la réalisation d'une recommandation de bonnes pratiques (RBP) sur la prise en charge des polypes de moins de

\footnotetext{
D. Heresbach $(\square)$

Unité d'endoscopie digestive, centre hospitalier de Cannes,

15 , avenue des Broussailles, CS 50008,

F-06414 Cannes, France

e-mail : d.heresbach@ch-cannes.fr
}

$5 \mathrm{~mm}$ ». Après un long silence, il nous a été répondu que «Le comité de validation des recommandations de bonne pratique (CVR) avait donné un avis défavorable au projet de RBP sur "prise en charge du polype rectocolique strictement inférieur à $6 \mathrm{~mm}$ " ». Le CVR a fondé son avis sur le fait que les polypes inférieurs ou égaux à $6 \mathrm{~mm}$ sont exceptionnellement cancéreux, avec un risque très faible pour les lésions synchrones de dysplasie de haut grade ou de cancer débutant et des données rassurantes sur le risque métachrone quand ils sont laissés sur place !

Après plusieurs demandes d'éclaircissement sur ce refus où un comité d'évaluation se substituait à une revue scientifique par les groupes de travail et de lecture constitués de professionnels, la commission des recommandations de bonne pratique de la HAS a botté dans les « $22 \mathrm{~m}$ » de l'INCa sous prétexte que le polype de moins de $5 \mathrm{~mm}$ pouvait être cancéreux ! - en toute concordance avec les conclusions de son CVR. Après un deuxième et troisième longs silences de l'INCa, les conclusions d'une conférence téléphonique étaient : « nous attendons notre feuille de route ». Ce n'est que 2,5 mois plus tard que le CA de la SFED a obtenu une réponse formelle : " faites une demande de subvention, hors appel d'offres pour que la SFED réalise un rapport d'expert » dans lequel la HAS ne sera pas partie prenante, ni ses membres impliqués, et qui ne sera pas labellisé par la HAS.

Dans un épisode parallèle, la HAS a encore fait preuve de sa pertinence en 2011 à propos de son implication dans le dossier du consensus formalisé d'expert sur « la sédation par les non-anesthésistes ». Réactivé en 2009 par la HAS et la SFAR, ce dossier a donné lieux pendant l'année 2010 à quatre réunions des groupes de travail et de lecture impliquant la SFAR, la SFED et la SNGE mais aussi d'autres sociétés savantes, de radiologie ou de pneumologie, pour ne citer que les présents ! Débouchant sur un texte de qualité, équilibré, dont les grandes lignes ont été discutées lors des journées de réflexion de la SFED en janvier 2011, la validation par les CA des sociétés savantes devait être obtenue pour sa diffusion et ensuite appropriation et/ou application par les 
établissements de santé qui en aurait besoin. Faute de nouvelles, alors que le CA de la SFED avait en mars 2011 approuvé les conclusions du texte proposé, la HAS s'est réfugiée après notre relance en mai 2011 derrière deux arguments :

- la HAS n'est pas promotrice, mais que partenaire !... pas de bon augure pour le dossier sur le polype de moins de $5 \mathrm{~mm}$ qui ne sera donc qu'un rapport d'expert de la SFED ;

- Le CVR n'a pas eu connaissance du texte et ne peut donc pas le labéliser, car la HAS ne dispose pas des déclarations d'intérêt de tous les experts du groupe de travail !

Ce curieux pilotage et situation invalide-t-il pour autant les textes rédigés entre professionnels et doit-on tout de même les publier... s'interroge le rédacteur en chef?

Alors qu'il est d'actualité de savoir si des recommandations nationales de la SFED demeurent indispensables ou s'il est plus simple et aussi valable médicalement de fonder notre pratique sur les recommandations et guidelines européennes (ESGE) ou anglo-saxonnes (ASGE), les manques et « absences » de nos agences ou instituts nationaux de santé apportent une réponse sur un plateau. Faute de moyens ou de responsabilité contractualisée, la dérive de 2011 démontre la limite de ces agences dont on peut s'interroger sur leur mode de fonctionnement (pour rester politiquement correct).

Ces deux aventures doivent entraîner une réflexion et apporter des conclusions sur l'indépendance et l'autonomie des recommandations professionnelles au-delà du pouvoir politico-social qui nomme ou mute les quelques « décideurs » de ces agences apparemment de moins en moins en phase avec le quotidien des professionnels de santé. Le malheur veut que par facilité ou impossibilité financière, pour que les professionnels, via leurs sociétés savantes, mènent des recommandations selon la méthodologie EBM ; faute de réflexion et d'organisation, seuls les textes des thématiques qui sont sous la coupe ou sélectionnés et publiés par les agences (et donc de ceux qui en nomment les décideurs) seront disponibles ! La gouverne de la réflexion médico-scientifique par le pouvoir politico-économique ou par ceux qui sont nommés pour en relayer les décisions, avec tous ses « rouages ou arrangements », est également un conflit d'intérêt, mais institutionnel qui devra « ou devrait » être déclaré par les signataires de ce type de décisions.

Plus globalement, cette réflexion doit nous inviter à mieux définir le terme " conflit d'intérêt ». Les définition et dénomination doivent être modifiées car, par exemple, le terme utilisé par les instituions est « DPI pour Déclaration Publique d'Intérêt », ce qui est déjà mieux que « conflits ». Ce dernier sous-entend que les textes ou décisions ont été orientés ou influencés par les intérêts de l'auteur alors qu'une « déclaration » est un avertissement ou une informa- tion du lecteur sans préjuger si les textes ou affirmations de l'auteur sont ou non objectifs ou influencés. Le terme « intérêt » doit être également modifié car il peut être compris comme « avoir intérêt à » mais également se limite (on le voit tous les jours) « aux intérêts financiers » (le terme se prête bien à cette confusion). Le mot « interférence » serait plus adapté car il permet de garder l'acronyme « DPI pour Déclaration Publique d'Interférence » et englobe plus largement d'autres interférences, morale, religieuse, politique, conjugale ou autres ! En effet, dans la définition du « préimprimé » de la HAS, on stigmatise l'époux ou l'épouse (qui est exclu[e] du projet de loi pour les DPI des parlementaires et homme ou femme politique !), mais c'est renier la valeur républicaine du PACS ou de tout autre forme de contrat ! Néanmoins, la DPI ne doit pas, comme souhaité par les politiques, ne concerner que la personne « déclarant la DPI», car la HAS n'a point besoin de connaître les propriétés, stock-option ou participation de nos épouse ou époux puisque l'interférence est implicite dans ce cas, à l'inverse de l'intérêt financier. Enfin, la durée ou le délai sur lequel porte la DPI ne trouve aucun fondement, car la mémoire est plus moins vive selon les personnes, sans compter que l'on peut perdre la mémoire des faits nouveaux et/ou anciens. La seule base objective serait une durée équivalente aux mandats, contrats ou pactes, sauf si l'interférence ou la gratification laisse une cicatrice éternelle. Cinq ans est le délai retenu par la HAS, mais il est vrai que le mandat suprême de nos politiques est de cinq ans ; la majorité des mandats dans notre profession ont une durée de deux à quatre ans, donc trois ans ne pourrait être ni une moyenne ni une médian, mais un choix. Ces quelques remarques ne devraient pas surcharger la HAS pour modifier son préimprimé qui, je l'espère, ne leur a pas pris trop de temps, temps qui aurait pu être mis à profit pour enregistrer les DPI des participants au CFE sur la SNA afin de se saisir leur CVR et publier un guide pour la communauté médicale... sauf à imaginer que la HAS ou d'autres avaient intérêt à ce que la sédation par les non-anesthésistes ou SNA avorte ! oui, il est temps de disposer des DPI de tous les acteurs.

Conflit d'intérêt : Le Pr D Heresbach a réalisé des missions ponctuelles d'expert auprès des laboratoires Norgine, Aptalis en 2010, et de la société MedPass, des laboratoires Norgine et Aptalis en 2011. Il a participé comme rédacteur en chef à un symposium organisé par Mauna Kea Technology en 2010 et Ella SA en 2011, et participera au CFE de 2012 avec le soutien de Wilson-Cook France. Il est coordonnateur de registre des polypes d'Ille et Vilaine (2009-2010) en partenariat à hauteur de $30 \%$ par les laboratoires Ferring SA et d'un STIC financé par l'INCa en 2009-2010 sur la coloscopie virtuelle. 